\title{
Exact Solutions of the Mass-Dependent Klein-Gordon Equation with the Vector Quark-Antiquark Interaction and Harmonic Oscillator Potential
}

\author{
M. K. Bahar ${ }^{1,2}$ and F. Yasuk ${ }^{1}$ \\ ${ }^{1}$ Department of Physics, Erciyes University, 38039 Kayseri, Turkey \\ ${ }^{2}$ Department of Physics, Karamanoglu Mehmetbey University, 70100 Karaman, Turkey \\ Correspondence should be addressed to F. Yasuk; yasuk@erciyes.edu.tr
}

Received 10 December 2012; Accepted 23 December 2012

Academic Editor: S. H. Dong

Copyright ( 2013 M. K. Bahar and F. Yasuk. This is an open access article distributed under the Creative Commons Attribution License, which permits unrestricted use, distribution, and reproduction in any medium, provided the original work is properly cited.

Using the asymptotic iteration and wave function ansatz method, we present exact solutions of the Klein-Gordon equation for the quark-antiquark interaction and harmonic oscillator potential in the case of the position-dependent mass.

\section{Introduction}

The movement of a particle with harmonic oscillations around an equilibrium position creates one of the most fundamental problems of physics. The basic structure of different systems such as vibration of the diatomic molecules, oscillations of atoms in crystal lattices, or nucleons in core is a harmonic oscillator problem [1]. Further, the quantum theory of electromagnetic fields is closely related to the examples of the harmonic oscillator $[2,3]$. When a particle is in a strong potential field, the relativistic effect must be considered. However, in relativistic and nonrelativistic quantum mechanics, many authors have adequately the harmonic oscillator problem $[4,5]$. But, it has never been investigated for relativistic particles with position-dependent mass (pdm). Quantum mechanical review of relativistic spin0 particles with pdm in the harmonic oscillator potential is very important in terms of understanding physical behavior of systems as the above-mentioned systems. The studies of quantum and relativistic quantum systems with pdm have received increasing attention in the literature. Systems with pdm have been found to be very useful in studying the physical and electronic properties of semiconductors, quantum wells and quantum dots, quantum liquids,
${ }^{3}$ He clusters, graded alloys, and semiconductor heterostructures [6]. To study the quasi-exactly solvable and exactly solvable nonrelativistic Schrödinger, relativistic Klein-Gordon and Dirac equations in the presence of pdm having a suitable mass distribution functions in one, three, and/or any arbitrary D-dimensional cases for different potentials have been used in different methods by many authors [7-21].

In this study, in addition to the examination of the harmonic oscillator potential, relativistic spin-0 particles with pdm have also been investigated in the quark-antiquark interaction potential. This type of potential and some of central potentials have recently been studied using different techniques [22-25]. The quark-antiquark interaction potential consists of harmonic, linear, and Coulomb potential terms. As we know, the quark-antiquark interaction potentials are a spherically symmetrical potential. The spherically symmetrical potential model also presents a good description of heavy quarkonium mass spectra such as charmonium and bottomonium. The interaction potentials for such systems are of a confining type called the Cornell potential. The Cornell potential consists of two terms, namely, the Coulomb and linear terms. The Coulomb term is responsible for the interaction at small distances and the linear term leads to the confinement. This type of interaction potential is also 
supported by lattice quantum chromodynamics calculations [26]. The quark-antiquark interaction has also been studied using the Coulomb term plus the power potential [27].

The organization of this paper is as follows. In the second section, the AIM is given shortly. In the third section, the general formalism of the Klein-Gordon equation for spin0 particles with pdm has been considered. The relativistic energy eigenvalues and corresponding eigenfunctions have been presented for the harmonic oscillator and quarkantiquark interaction potentials preserved in the fourth and fifth section, respectively. Finally, conclusions are given in the last section.

\section{Basic Equations of the AIM}

We briefly outline the AIM here; the details can be found in [28-30]. The AIM was proposed to solve second-order differential equations of the form:

$$
y^{\prime \prime}=\lambda_{0}(x) y^{\prime}+s_{0}(x) y,
$$

where $\lambda_{0}(x) \neq 0$ and $s_{0}(x)$ are in $C_{\infty}(a, b)$, and these variables are sufficiently differentiable. The differential equation (1) has a general solution as follows:

$$
\begin{aligned}
y(x) & \\
= & \exp \left(-\int^{x} \alpha d x^{\prime}\right) \\
& \times\left[C_{2}+C_{1} \int^{x} \exp \left(\int^{x^{\prime}}\left[\lambda_{0}\left(x^{\prime \prime}\right)+2 \alpha\left(x^{\prime \prime}\right)\right] d x^{\prime \prime}\right) d x^{\prime}\right]
\end{aligned}
$$

if $n>0$, for sufficiently large $n$,

$$
\frac{s_{n}}{\lambda_{n}}=\frac{s_{n-1}}{\lambda_{n-1}}=\alpha_{k}
$$

where

$$
\begin{aligned}
& \lambda_{n}(x)=\lambda_{n-1}^{\prime}(x)+s_{n-1}(x)+\lambda_{0}(x) \lambda_{n-1}(x), \\
& s_{n}(x)=s_{n-1}^{\prime}(x)+s_{0}(x) \lambda_{n-1}(x), \quad n=1,2,3, \ldots
\end{aligned}
$$

The termination condition of the method together with (4) can be also written as follows:

$$
\delta(x)=\lambda_{n+1}(x) s_{n}(x)-\lambda_{n}(x) s_{n+1}(x)=0 .
$$

For a given potential, the idea is to convert the relativistic wave equation to the form of (1). Then, $s_{0}$ and $\lambda_{0}$ are determined and $s_{n}$ and $\lambda_{n}$ parameters are calculated. The energy eigenvalues are obtained by the termination condition given by (5). However, the exact eigenfunctions can be derived from the following wave function generator:

$$
y_{n}(x)=C_{2} \exp \left(-\int^{x} \alpha_{k} d x^{\prime}\right),
$$

where $n=0,1,2, \ldots$ and $k$ is the iteration step number, and it is greater than $n$.

\section{Formalism of the Klein-Gordon Equation with Pdm}

In the relativistic quantum mechanics, for spin-0 particles with pdm, the Klein-Gordon equation is defined as follows:

$$
\begin{gathered}
\nabla^{2} \psi(r)+\frac{1}{\hbar^{2} c^{2}}\left[\left(E_{n l}-V(r)\right)^{2}-\left(m(r) c^{2}+S(r)\right)^{2}\right] \psi(r)=0, \\
\nabla^{2}=\sum_{i=1}^{3} \frac{\partial^{2}}{\partial x_{i}^{2}},
\end{gathered}
$$

where $V(r)$ and $S(r)$ are Lorentz vector and scalar potential, respectively, $m(r)$ is mass function, and $E_{n l}$ is the energy of particle. Let us decompose the radial wave function $\psi(r)$ as follows:

$$
\psi(r)=\frac{u(r)}{r} Y_{m}^{l}(\widehat{r}),
$$

where $u(r)$ is the radial wave function and $Y_{m}^{l}(\widehat{r})$ is the angular dependent spherical harmonics, and this reduces (8) into the following Schrödinger-like equation with positiondependent mass:

$$
\begin{aligned}
& \frac{d^{2} u(r)}{d r^{2}}+\frac{1}{\hbar^{2} c^{2}} \\
& \times\left(\left[E_{n l}-V(r)\right]^{2}-\left[m(r) c^{2}+S(r)\right]^{2}-\frac{l(l+1)}{r^{2}}\right) \\
& \quad \times u(r)=0 .
\end{aligned}
$$

\section{In Case of Harmonic Oscillator Potential}

4.1. The Eigenvalues. In case of harmonic oscillator to investigate spin-0 particles with pdm, we should solve (9). In this solution, we use atomic units $\hbar=c=1$. However, in (9), we prefer to use mass function similar to type of harmonic oscillator potential as follows:

$$
m(r)=m_{0}+\frac{1}{2} k r^{2}
$$

where $m_{0}$ and $k$ are positive constants. The selection as in (10) of position dependent mass function is more suitable both physically and mathematical. Already, in physical applications, the position-dependent mass creates a new effective potential by shifting potential profile of the system.

In this study, in the absence of scalar potential, vector harmonic oscillator potential is defined as

$$
V(r)=\frac{1}{2} m(r) \omega^{2} r^{2}
$$

where $\omega=\sqrt{k / m(r)}$ is the angular frequency and $k$ is elastic coefficient. 
In the presence of vector potential and by taking $\hbar=c=1$, if (10) and (11) are inserted into (9), it is obtained that

$$
\left[\frac{d^{2}}{d r^{2}}+\xi_{0}-\xi_{1} r^{2}-\frac{\xi_{2}}{r^{2}}\right] u(r)=0,
$$

where $\xi_{0}=E_{n l}^{2}-m_{0}^{2}, \xi_{1}=k\left(E_{n l}-m_{0}\right), \xi_{2}=l(l+1)$.

In (12), while $r$ approaches zero and infinite, solving of (12) are $r^{(1 / 2)\left(1+\sqrt{1+4 \xi_{2}}\right)}, e^{-r^{2} \sqrt{\xi_{1}} / 2}$, respectively. Therefore, the reasonable physical wave function is proposed as follows:

$$
u_{n l}(r)=r^{(1 / 2)\left(1+\sqrt{1+4 \xi_{2}}\right)} e^{-r^{2} \sqrt{\xi_{1}} / 2} f_{n l}(r) .
$$

Substituting (13) into (12), we have second-order homogeneous linear differential equation:

$$
\begin{aligned}
f^{\prime \prime}(r)= & \left(-\frac{1+\sqrt{1+4 \xi_{2}}}{r}+2 r\right) f^{\prime}(r) \\
& -\left(\xi_{0}-\sqrt{\xi_{1}}\left(2+\sqrt{1+4 \xi_{2}}\right)\right) f(r) .
\end{aligned}
$$

Defining a new variable $z=\sqrt{\xi_{1}} r^{2}$, so doing, we have solvable differential equation by AIM as follows:

$$
\begin{aligned}
f^{\prime \prime}(z)= & \left(1-\frac{2+\sqrt{1+4 \xi_{2}}}{2 z}\right) f^{\prime}(z) \\
& +\frac{\sqrt{\xi_{1}}\left(2+\sqrt{1+4 \xi_{2}}\right)-\xi_{0}}{4 \sqrt{\xi_{1}} z} f(z) .
\end{aligned}
$$

By comparing (15) with (1), $\lambda_{0}(z)$ and $s_{0}(z)$ values are obtained, and using (4) we calculate $\lambda_{n}(z)$ and $s_{n}(z)$. In this way,

$$
\begin{aligned}
\lambda_{0}(z)= & \left(1-\frac{\left.2+\sqrt{1+4 \xi_{2}}\right)}{2 z}\right) \\
s_{0}(z)= & \frac{\sqrt{\xi_{1}}\left(2+\sqrt{1+4 \xi_{2}}\right)-\xi_{0}}{4 \xi_{1} z}, \\
\lambda_{1}(z)= & \left(4 \xi_{1}\left(2-2 z+\sqrt{1+4 \xi_{2}}\right)^{2}\right. \\
& +4 \sqrt{\xi_{1}}\left(-2 z \xi_{0}+2 \sqrt{\xi_{1}}\left(2+\sqrt{1+4 \xi_{2}}\right)\right) \\
& \left.+z \sqrt{\xi_{1}}\left(2+\sqrt{1+4 \xi_{2}}\right)\right) \times\left(16 z^{2} \xi_{1}\right)^{-1} \\
s_{1}(z)= & \left(2-\sqrt{\xi_{1}}\left(2+\sqrt{1+4 \xi_{2}}\right)\right) \\
& \left.\times\left(4 \sqrt{\xi_{1}}+2 \sqrt{\xi_{1}}\left(2-2 z+\sqrt{1+4 \xi_{2}}\right)\right)\right) \\
\times & \left(16 z^{2} \xi_{1}\right)^{-1}
\end{aligned}
$$

Combining these results obtained by the AIM with quantization condition given by (5) yields

$$
\begin{aligned}
s_{0} \lambda_{1}-s_{1} \lambda_{0} & =0 \Longrightarrow \xi_{00} \\
= & \frac{1}{2} \sqrt{\xi_{1}}\left(2+\sqrt{1+4 \xi_{2}}\right), \quad \text { for } n=0 \\
s_{1} \lambda_{2}-s_{2} \lambda_{1}=0 \Longrightarrow \xi_{01} & \\
= & \frac{1}{2} \sqrt{\xi_{1}}\left(6+\sqrt{1+4 \xi_{2}}\right), \quad \text { for } n=1 \\
s_{2} \lambda_{3}-s_{3} \lambda_{2}=0 & \Longrightarrow \xi_{02} \\
= & \frac{1}{2} \sqrt{\xi_{1}}\left(10+\sqrt{1+4 \xi_{2}}\right), \quad \text { for } n=2
\end{aligned}
$$

If the set of equations (17a), (17b), and (17c) are generalized, the indirect energy eigenvalues statement turns out to be

$$
\xi_{0 n}=\frac{1}{2} \sqrt{\xi_{1}}\left(4 n+2+\sqrt{1+4 \xi_{2}}\right)
$$

When (18) and $\xi_{0}=E_{n l}^{2}-m_{0}^{2}$ are compared, it is found for energy eigenvalues that

$$
E_{n l}^{2}=m_{0}^{2}+\frac{\sqrt{k\left(E_{n l}-m_{0}\right)}}{2}(4 n+2+\sqrt{1+4 l(l+1)}) .
$$

4.2. The Eigenfunctions. The exact eigenfunctions can be derived from the following generator:

$$
f_{n}(z)=C_{2} \exp \left(-\int^{z} \alpha_{k} d z^{\prime}\right) .
$$

Using (3) and (20), the eigenfunctions are obtained as follows:

$$
\begin{gathered}
f_{0}(z)=1 \\
f_{1}(z)=\left(z-\frac{\left(2+\sqrt{1+4 \xi_{2}}\right)}{2}\right) \\
f_{2}(z)=\left(\frac{\left.2+\sqrt{1+4 \xi_{2}}\right)\left(1+\frac{2+\sqrt{1+4 \xi_{2}}}{2}\right)}{2}-2\left(1+\frac{2+\sqrt{1+4 \xi_{2}}}{2}\right) z+z^{2}\right.
\end{gathered}
$$

Finally, the following general formula for the exact solutions $f_{n}(z)$ is acquired as

$$
\begin{aligned}
f_{n}(z)= & (-1)^{n}\left(\frac{2+\sqrt{1+4 \xi_{2}}}{2}\right)_{n} \\
& \times{ }_{1} F_{1}\left(-n, \frac{2+\sqrt{1+4 \xi_{2}}}{2} ; z\right) .
\end{aligned}
$$


Hence, we write the total radial wavefunction as follows:

$$
\begin{aligned}
u_{n l}(r)= & N r^{(1 / 2)\left(1+\sqrt{1+4 \xi_{2}}\right)} e^{-r^{2} \sqrt{\xi_{1}} / 2}\left(\frac{2+\sqrt{1+4 \xi_{2}}}{2}\right)_{n} \\
& \times{ }_{1} F_{1}\left(-n, \frac{2+\sqrt{1+4 \xi_{2}}}{2} ; \xi_{1} r^{2}\right),
\end{aligned}
$$

where $N$ is normalization constant.

\section{In Case of Quark-Antiquark Interaction Potential}

5.1. The Eigenvalues and Corresponding Eigenfunctions. In this section, we present the solution of the Klein-Gordon equation for the quark-antiquark interaction potential. This potential is defined as

$$
V(r)=a r^{2}+b r-\frac{c}{r}, \quad a>0
$$

where $a, b$, and $c$ are constants. In atomic units $\hbar=c=1$, we solve (9) in the absence of scalar potential. Consider the position-dependent mass function:

$$
m(r)=m_{0}+a r^{2}+b r-\frac{c}{r} .
$$

On substituting (24) and (25) into (9), we find that

$$
\left[\frac{d^{2}}{d r^{2}}+\varepsilon-\varepsilon_{0} r^{2}-\varepsilon_{1} r+\frac{\varepsilon_{2}}{r}-\frac{l(l+1)}{r^{2}}\right] u_{l}(r)=0
$$

where $\varepsilon=E_{n l}^{2}-m_{0}^{2}, \varepsilon_{0}=2 a\left(E_{n l}+m_{0}\right), \varepsilon_{1}=2 b\left(E_{n l}+m_{0}\right)$, and $\varepsilon_{2}=2 c\left(E_{n l}+m_{0}\right)$.

To solve (26), applying an ansatz to the radial wavefunction $u_{l}(r)$,

$$
u_{l}(r)=e^{\alpha r+(\gamma / 2) r^{2}} \sum_{n=0} a_{n} r^{n+\delta}
$$

If (27) is inserted into (26), it is obtained that

$$
\begin{aligned}
& \sum_{n=0} a_{n} \underbrace{[\gamma+2 \gamma(n+\delta)+\varepsilon]}_{A_{n}} r^{n+\delta}+\sum_{n=0} a_{n} \underbrace{\left[2 \alpha \gamma-\varepsilon_{1}\right]}_{2 \alpha \gamma=\varepsilon_{1}} r^{n+\delta+1} \\
& +\sum_{n=0} a_{n} \underbrace{\left[\alpha^{2}-\varepsilon_{0}+\gamma^{2}\right]}_{\varepsilon_{0}=\alpha^{2}+\gamma^{2}} r^{n+\delta+2}+\sum_{n=0} a_{n} \underbrace{\left[2 \alpha(n+\delta)+\varepsilon_{2}\right]}_{B_{n}} r^{n+\delta-1} \\
& +\sum_{n=0} a_{n} \underbrace{[(n+\delta)(n+\delta-1)-l(l+1)]}_{C_{n}} r^{n+\delta-2}=0, \\
& \varepsilon_{1}=2 \alpha \gamma, \quad \varepsilon_{0}=\alpha^{2}+\gamma^{2}
\end{aligned}
$$

$\alpha$ and $\gamma$ can be obtained with the help of (29). Then, $\alpha=$ $\left(-\sqrt{\varepsilon_{0}+\varepsilon_{1}}-\sqrt{\varepsilon_{0}-\varepsilon_{1}}\right) / 2$ and $\gamma=\left(-\sqrt{\varepsilon_{0}+\varepsilon_{1}}+\sqrt{\varepsilon_{0}-\varepsilon_{1}}\right) / 2$. Editing (28),

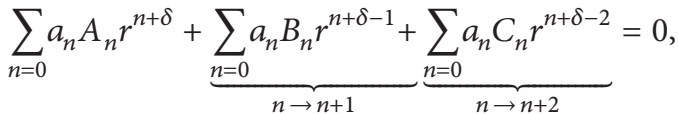

$$
\begin{aligned}
& \underbrace{\left(a_{0} B_{0}+a_{1} C_{1}\right)}_{0} r^{\delta-1}+a_{0} \underbrace{C_{0}}_{0} r^{\delta-2} \\
& +\sum_{n=0} \underbrace{\left(a_{n} A_{n}+a_{n+1} B_{n+1}+a_{n+2} C_{n+2}\right)}_{0} r^{n+\delta}=0 .
\end{aligned}
$$

In (31), if the first nonvanishing coefficient is $a_{0} \neq 0, C_{0}$ should be equal to zero:

$$
C_{0}=\delta(\delta-1)-l(l+1)=0 .
$$

We choose $\delta=l+1$ as a physically acceptable solution from (32). Moreover, if the $p$ th nonvanishing coefficient is $a_{p} \neq 0$, but $a_{p+1}=a_{p+2}=\cdots=0$, then, from (31), it has to be $A_{p}=0$. At that rate,

$$
\gamma+2 \gamma(p+\delta)+\varepsilon=0
$$

Using together (29) and (33), we obtain the energy eigenvalues. Namely,

$$
\frac{b\left(E_{n l}+m_{0}\right)}{\alpha}+\frac{2 b\left(E_{n l}+m_{0}\right)}{\alpha}(p+l+1)+E_{n l}^{2}-m_{0}^{2}=0 .
$$

$A_{n}, B_{n}$, and $C_{n}$ must satisfy the determinant relation for a nontrivial solution:

$$
\operatorname{det}\left|\begin{array}{cccccc}
B_{0} & C_{1} & \ldots & \ldots & \ldots & 0 \\
A_{0} & B_{1} & C_{2} & \cdots & \ldots & . \\
\vdots & \vdots & \vdots & \ddots & \vdots & \vdots \\
0 & 0 & 0 & 0 & A_{p-1} & B_{p}
\end{array}\right|=0
$$

In order to appreciate this method, we present the exact solutions for the cases $p=0,1$ as follows.

If $p=0, \operatorname{det}\left|B_{0}\right|=0$ and $B_{0}=0$. So,

$$
B_{0}=0 \Longrightarrow 2\left(E_{0}+m_{0}\right) c=-2(l+1) \alpha .
$$

We will obtain energy eigenvalues by using (34). But, we cannot ignore (36) Because it is a restriction on the parameters of the potential and the $l$ quantum number.

The corresponding eigenfunction for $p=0$ is given as

$$
\begin{array}{r}
u_{l}^{0}(r)=a_{0} \exp \left[-\frac{\sqrt{\varepsilon_{0}+\varepsilon_{1}}+\sqrt{\varepsilon_{0}-\varepsilon_{1}}}{2} r\right. \\
\left.-\frac{\sqrt{\varepsilon_{0}+\varepsilon_{1}}-\sqrt{\varepsilon_{0}-\varepsilon_{1}}}{4} r^{2}\right] r^{\delta},
\end{array}
$$

where $a_{0}$ is the normalization constant. 
If $p=1, \operatorname{det}\left|\begin{array}{ll}B_{0} & C_{1} \\ A_{0} & B_{1}\end{array}\right|=0$. In this case, it is obtained that

$\underbrace{\left(\frac{\varepsilon_{1}}{2 \alpha}+\frac{\varepsilon_{1}}{\alpha}(l+1)+\varepsilon\right) 2(l+1)-\left(\varepsilon_{2}+2 \alpha(l+1)\right)\left(\varepsilon_{2}+2 \alpha(l+2)\right)}_{\text {for } E_{1}}$

$=0$,

which is a restriction on the parameters of the potential and the angular momentum quantum number.

The corresponding eigenfunction for $p=1$ is given as

$$
\begin{array}{r}
u_{l}^{1}(r)=\left(a_{0}+a_{1} r\right) \exp \left[-\frac{\sqrt{\varepsilon_{0}+\varepsilon_{1}}+\sqrt{\varepsilon_{0}-\varepsilon_{1}}}{2} r\right. \\
\left.-\frac{\sqrt{\varepsilon_{0}+\varepsilon_{1}}-\sqrt{\varepsilon_{0}-\varepsilon_{1}}}{4} r^{2}\right] r^{\delta},
\end{array}
$$

where $a_{0}$ is the normalization constant.

Following in this way, we can generate a class of exact solutions by setting $p=1,2, \ldots$. Generally, if $a_{p} \neq 0$, but $a_{p+1}=a_{p+2}=\cdots=0$. So, the energy eigenvalues $E_{p}$ is obtained by using (34). The corresponding eigenfunction is

$$
\begin{aligned}
u_{l}^{p}(r)= & \left(a_{0}+a_{1} r+\cdots+a_{p} r^{p}\right) \\
& \times \exp \left[-\frac{\sqrt{\varepsilon_{0}+\varepsilon_{1}}+\sqrt{\varepsilon_{0}-\varepsilon_{1}}}{2} r\right. \\
& \left.-\frac{\sqrt{\varepsilon_{0}+\varepsilon_{1}}-\sqrt{\varepsilon_{0}-\varepsilon_{1}}}{4} r^{2}\right] r^{\delta},
\end{aligned}
$$

where $a_{0}, a_{1}, \ldots, a_{p}$ are normalization constants.

\section{Conclusions}

This paper has presented a different approach, the AIM, to calculate the bound state solutions of the relativistic KleinGordon with the harmonic oscillator potential in the case of the pdm. For arbitrary quantum number $l$ state, we have exactly obtained the energy eigenvalues and corresponding eigenfunctions for the case of mass function by AIM. The advantage of the AIM is that it gives the eigenvalues directly by transforming the second-order differential equation into a form of $y^{\prime \prime}=\lambda_{0}(r) y^{\prime}+s_{0}(r) y$. The exact wavefunctions are easily constructed by iterating the values of $s_{0}$ and $\lambda_{0}$. The method presented in this study is general and worth extending to the solution of other interactions. For the quarkantiquark interaction potential, to solve Klein-Gordon equation with pdm, we have used wave function ansatz method. While using this method, the most important factor to be considered is a corresponding restriction on the parameters of the quark-antiquark potential and the $l$ quantum number.

\section{Acknowledgment}

The authors wish to thank Professor Shi-Hai Dong.

\section{References}

[1] D. Faiman and A. W. Hendry, "Harmonic-oscillator model for baryons," Physical Review, vol. 173, no. 5, pp. 1720-1729, 1968.

[2] H. R. Lewis and W. B. Riesenfeld, "An exact quantum theory of the time-dependent harmonic oscillator and of a charged particle in a time-dependent electromagnetic field," Journal of Mathematical Physics, vol. 10, no. 8, 16 pages, 1969.

[3] L. Brey, N. F. Johnson, and B. I. Halperin, "Optical and magnetooptical absorption in parabolic quantum wells," Physical Review B, vol. 40, no. 15, pp. 10647-10649, 1989.

[4] Q. Wen-Chao, "Bound states of the Klein-Gordon and Dirac equations for scalar and vector harmonic oscillator potentials," Chinese Physics, vol. 11, no. 8, p. 757, 2002.

[5] B. C. Qian and J. Y. Zeng, The Choiceness and Anatomy for Problems of Quantum Mechanics, vol. 2, Science Press, Beijing, China, 1999.

[6] G. Levai and O. Özer, "An exactly solvable Schrödinger equation with finite positive position-dependent effective mass," Journal of Mathematical Physics, vol. 51, no. 9, Article ID 092103, 13 pages, 2010.

[7] S. M. Ikhdair and R. Sever, "Solutions of the spatially-dependent mass Dirac equation with the spin and pseudospin symmetry for the Coulomb-like potential," Applied Mathematics and Computation, vol. 216, no. 2, pp. 545-555, 2010.

[8] A. D. Alhaidari, "Relativistic extension of the complex scaling method," Physical Review A, vol. 75, no. 4, Article ID 042707, 11 pages, 2007.

[9] A. D. Alhaidari, H. Bahlouli, A. Al Hasan, and M. S. Abdelmonem, "Relativistic scattering with spatially dependent effective mass in the dirac equation," Physical Review A, vol. 75, no. 6, Article ID 062711, 14 pages, 2007.

[10] A. Alhaidari, "Solution of the Dirac equation with positiondependent mass in the Coulomb field," Physics Letters A, vol. 322, no. 1-2, pp. 72-77, 2004.

[11] A. de Souza Dutra and C.-S. Jia, "Classes of exact Klein-Gordon equations with spatially dependent masses: regularizing the one-dimensional inversely linear potential," Physics Letters A, vol. 352, no. 6, pp. 484-487, 2006.

[12] I. O. Vakarchuk, "The Kepler problem in Dirac theory for a particle with position-dependent mass," Journal of Physics A: Mathematical and General, vol. 38, p. 4727, 2005.

[13] C.-S. Jia, T. Chen, and L.-G. Cui, "Approximate analytical solutions of the Dirac equation with the generalized PöschlTeller potential including the pseudo-centrifugal term," Physics Letters A, vol. 373, pp. 1621-1626, 2009.

[14] C.-S. Jia and A. de Souza Dutra, "Extension of PT-symmetric quantum mechanics to the Dirac theory with positiondependent mass," Annals of Physics, vol. 323, no. 3, pp. 566-579, 2008.

[15] L. Dekar, L. Chetouani, and T. F. Hammann, "An exactly soluble Schrödinger equation with smooth position-dependent mass," Journal of Mathematical Physics, vol. 39, no. 5, 13 pages, 1998.

[16] X.-L. Peng, J.-Y. Liu, and C.-S. Jia, "Approximation solution of the Dirac equation with position-dependent mass for the generalized Hulthén potential," Physics Letters A, vol. 352, no. 6, pp. 478-483, 2006.

[17] Y. Xu, S. He, and C. S. Jia, "Approximate analytical solutions of the Dirac equation with the Pöschl-Teller potential including the spin-orbit coupling term," Journal of Physics A: Mathematical and Theoretical, vol. 41, no. 25, Article ID 255302, 2008. 
[18] R. Koc and M. Koca, "A systematic study on the exact solution of the position dependent mass Schrödinger equation," Journal of Physics A: Mathematical and General, vol. 36, no. 29, p. 8105, 2003.

[19] I. O. Vakarchuk, "The Kepler problem in Dirac theory for a particle with position-dependent mass," Journal of Physics A: Mathematical and Theoretical, vol. 38, no. 21, p. 4727, 2005.

[20] Y. Xu, S. He, and C. S. Jia, "Approximate analytical solutions of the Dirac equation with the Pöschl-Teller potential including the spin-orbit coupling term," Journal of Physics A: Mathematical and Theoretical, vol. 41, no. 25, Article ID 255302, 2008.

[21] A. Arda, R. Sever, and C. Tezcan, "Approximate analytical solutions of the Klein-Gordon equation for the Hulthén potential with the position-dependent mass," Physica Scripta, vol. 79, no. 1, Article ID 015006, 2009.

[22] R. Kumar and F. Chand, "Series solutions to the $N$-dimensional radial Schrödinger equation for the quark-antiquark interaction potential," Physica Scripta, vol. 85, no. 5, Article ID 055008, 2012.

[23] A. A. Rajabi, "Hypercentral constituent quark model and isospin for the baryon static properties," Journal of Sciences, Islamic Republic of Iran, vol. 16, pp. 73-79, 2005.

[24] S. H. Dong, "Exact solutions of the two dimensional Schrödinger equation with certain central potentials," International Journal of Theoretical Physics, vol. 39, no. 4, 2000.

[25] S. H. Dong, "A new approach to the relativistic Schrödinger equation with central potential: Ansatz method," International Journal of Theoretical Physics, vol. 40, no. 2, 2001.

[26] C. Alexandrou, P. de Forcrand, and O. Jahn, “The ground state of three quarks," Nuclear Physics B, vol. 119, pp. 667-669, 2003.

[27] B. Patel and P. C. Vinodkumar, "Properties of $Q \bar{Q}(Q \in b, c)$ mesons in Coulomb plus power potential $\left(\mathrm{CPP}_{v}\right)$," Journal of Physics G: Nuclear and Particle Physics, vol. 36, no. 3, Article ID 035003, 2009.

[28] H. Ciftci, R. L. Hall, and N. Saad, "Asymptotic iteration method for eigenvalue problems," Journal of Physics A: Mathematical and General, vol. 36, no. 47, pp. 11807-11816, 2003.

[29] H. Ciftci, R. L. Hall, and N. Saad, "Construction of exact solutions to eigenvalue problems by the asymptotic iteration method," Journal of Physics A: Mathematical and General, vol. 38, no. 5, pp. 1147-1155, 2005.

[30] H. Ciftci, R. L. Hall, and N. J. Saad, "Iterative solutions to the Dirac equation," Physical Review A, vol. 72, Article ID 022101, 7 pages, 2005. 

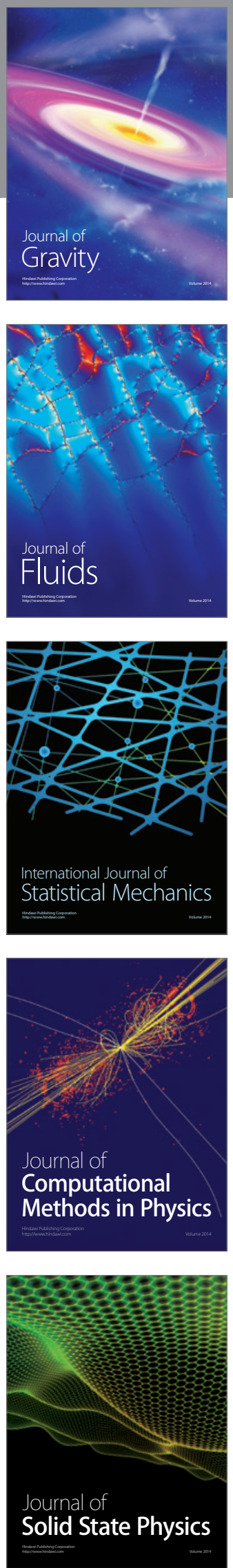

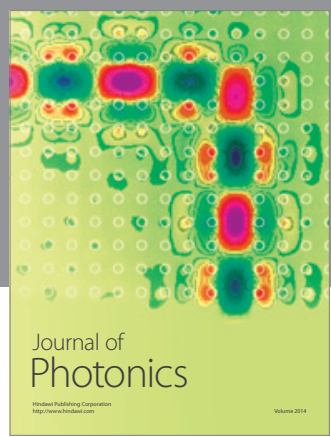

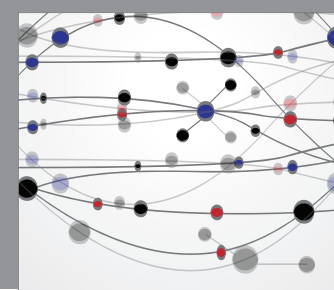

The Scientific World Journal

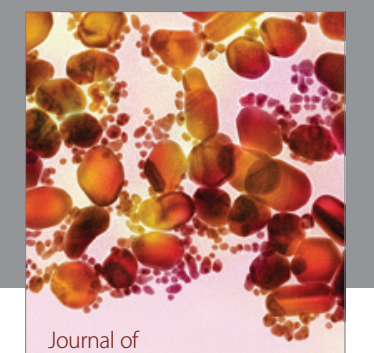

Soft Matter
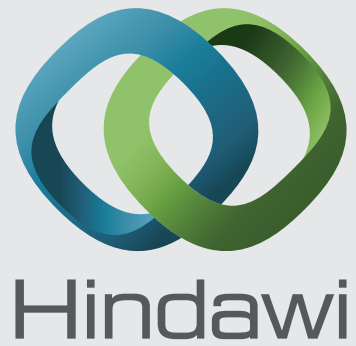

Submit your manuscripts at

http://www.hindawi.com
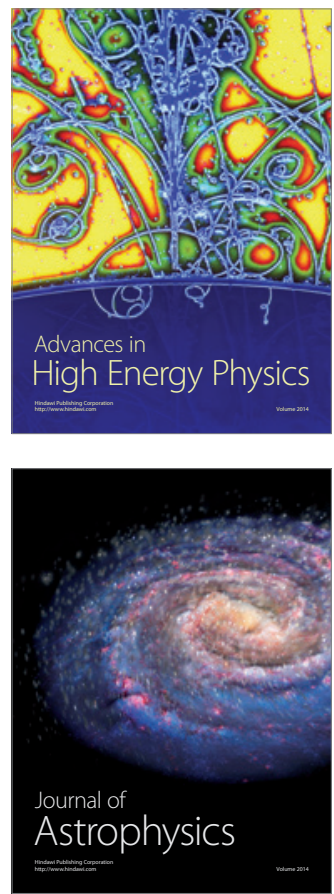
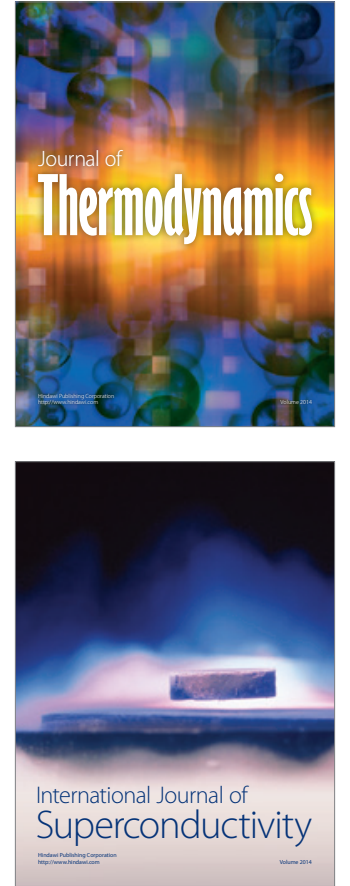
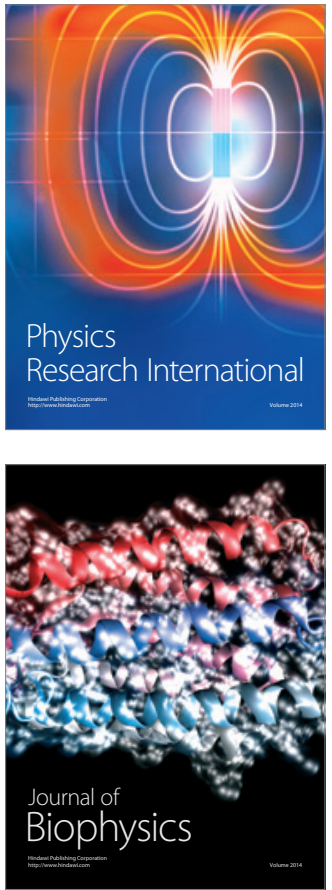
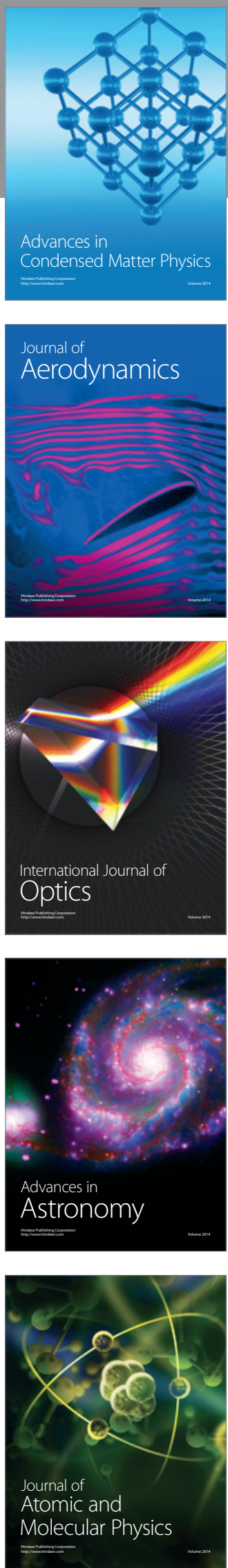